\title{
Gestión de la Exposición Laboral a Ruido en el Centro de Transferencia Tecnológica para la Capacitación e Investigación en Control de Emisiones Vehiculares (CCICEV) de la Escuela Politécnica Nacional
}

\author{
Carrera, Gisela $^{1}{ }^{*}$ (D) ; Salgado, Francisco ${ }^{1}$ (D) ; Villacis, William ${ }^{1}$ \\ ${ }^{1}$ Escuela Politécnica Nacional, Quito, Ecuador
}

\begin{abstract}
Resumen: El objetivo de esta investigación radica en: identificar los factores de riesgo que pueden afectar la salud auditiva del personal del CCICEV de la Escuela Politécnica Nacional, evaluar los riesgos y proponer las medidas de control que permitan el cumplimiento de la normativa vigente respecto a la exposición laboral de ruido en el Ecuador. En el CCICEV, se llegó a determinar que existe sobreexposición laboral a ruido, ya que luego de realizar la evaluación a riesgo físico, el nivel de presión sonora diario equivalente ponderado en A (Laeqd) en el área técnica, sobrepasa los $85 \mathrm{dBA}$ y en el área administrativa se encuentra sobre los $70 \mathrm{dBA}$. Por ello, fueron propuestas medidas de control para la fuente, el medio y el receptor, de acuerdo con la jerarquía de control de ruido de la norma ISO 45001; siendo estas: para la fuente de enfriamiento, una cabina de insonorización; para el medio, paneles de absorción acústica, así como un revestimiento acústico en la fachada frontal de las oficinas; como medidas administrativas, se proponen pausas pasivas o activas en lugares libres de ruido, apagar las fuentes de ruido si no se las necesita e informar a los colegas de trabajo si se va a realizar una actividad ruidosa; además se recomienda realizar un plan general de seguridad y salud en el trabajo donde prevalezca la vigilancia de la salud, la formación e información al igual que la debida señalización, y para el receptor del área técnica, se propone el uso de equipo de protección auditiva.
\end{abstract}

Palabras clave: Ruido, Riesgo Físico, Exposición Laboral, Higiene Industrial, Salud Auditiva.

\section{Management of Occupational Exposure to Noise at the Technology Transfer Center for Training and Research in Vehicle Emission Control (CCICEV) of the Escuela Politécnica Nacional}

\begin{abstract}
The objective of this research is to: identify the risk factors that may affect the hearing health of the CCICEV staff of the Escuela Politécnica Nacional, evaluate the risks and propose control measures that allow compliance with current regulations regarding the occupational noise exposure in Ecuador. The CCICEV determined that there is occupational overexposure to noise, since after carrying out the physical risk assessment, the equivalent daily A-weighted sound pressure level (Laeqd) in the technical area exceeds $85 \mathrm{dBA}$ and in the administrative area is over $70 \mathrm{dBA}$. For this reason, control measures were proposed for the source, the medium and the receiver, according to the noise control hierarchy of the ISO 45001 standard; being included: for the cooling source, a soundproofing cabin; for the middle, acoustic absorption panels, as well as an acoustic cladding on the front façade of the offices; As administrative measures, passive or active breaks are proposed in places free of noise, turning off noise sources if they are not needed and alerting co-workers if noisy activity is going to take place; In addition, it is recommended to carry out a general plan of health and safety at work where health surveillance, training and information prevail, along with proper signaling, and for the recipient of the technical area, the use of hearing protection equipment is proposed.
\end{abstract}

Keywords: Noise, Physical Risk, Occupational Exposure, Industrial Hygiene, Hearing Health.

\section{INTRODUCCIÓN}

Actualmente el avance tecnológico se encuentra ligado a la industria, permite que esta, crezca a pasos agigantados, y, lo que parece un beneficio, trae consigo daños colaterales; siendo uno de ellos el ruido, este afecta a la salud de las personas que se encuentran expuestas y más aún en condiciones laborales (Suter, 2012).

El ruido origina la pérdida progresiva de la capacidad auditiva y cada vez es mayor el número de personas afectadas. Se 
conoce además, que el ruido es un problema desde hace aproximadamente 2000 años (Baraza et al, 2014).

El ruido laboral es subestimado, dado que, los resultados de este se presentan luego de largos períodos de exposición, sus alertas son imperceptibles y cuando ya han sido identificadas, pueden haberse convertido en enfermedades profesionales donde los daños son irreparables (Suter, 2012).

El ruido está conformado por una serie de sonidos agrupados y no coordinados entre sí, estos sonidos no son entendibles, y producen sensaciones desagradables al oído, además interfieren con las actividades humanas (Falagán, 2008).

El intervalo de presión sonora o umbral auditivo que una persona con oídos sanos puede percibir y tolerar se encuentra entre $20 \times 10^{-6} \mathrm{~Pa}$ y $200 \mathrm{~Pa}$, si el valor fuera superior a este último, la persona se encontraría en un umbral doloroso que le provocaría lesiones irreversibles. Debido a que el oído responde de forma logarítmica con relación al ruido de un estímulo aplicado, las medidas acústicas se representan mediante una expresión logarítmica, de tal forma que, puedan llegar a ser manejables y fáciles de interpretar, tal es el caso del nivel de presión sonora representado en la Ecuación 1 (Falagán, 2008):

$$
L_{p}=10 x \log \left(\frac{P_{e f}}{P_{0}}\right)^{2}
$$

Donde:

Lp es el nivel de presión sonora [dB].

Pef es la presión eficaz que caracteriza a la onda [Pa].

Po es la presión de referencia $[\mathrm{Pa}]$.

De acuerdo con la NTP 270 "Evaluación de la exposición al ruido. Determinación niveles representativos", se considera cuatro tipos de ruido: el ruido estable, periódico, aleatorio y de impacto.

El ruido estable es considerado constante, ya que, la diferencia entre el valor máximo y mínimo en el nivel de presión sonora ponderada en $\mathrm{A}$ debe ser inferior a $5 \mathrm{~dB}$, para el ruido periódico esta diferencia debe ser igual o superior a $5 \mathrm{~dB}$ y debe poseer una cadencia cíclica. De igual forma que el caso anterior, para el ruido aleatorio, su diferencia puede ser superior o igual a $5 \mathrm{~dB}$ pero a su vez el nivel de presión sonora ponderada en A debe variar de forma aleatoria con el tiempo. En el en el caso del ruido de impacto no tiene similitud con ninguno de los casos anteriores ya que su nivel de presión sonora decrece exponencialmente en función del tiempo, siendo su duración menor a un segundo (INSHT NTP 270, s/f).

El ruido no desencadena los mismos efectos en todos los trabajadores, por lo tanto las pérdidas auditivas causadas por el ruido dependen de varios factores como:

$\begin{array}{ll}\text { - } & \text { Intensidad de ruido } \\ \text { - } & \text { Rango de frecuencias } \\ \text { - } & \text { Exposición diaria } \\ \text { - } & \text { Exposición prolongada } \\ & \text { Tipo de ruido }\end{array}$

\author{
- $\quad$ Susceptibilidad de cada trabajador \\ - Género \\ - $\quad$ Edad \\ - Afecciones previas del oído \\ - Eficiencia de métodos de protección auditiva \\ adoptados. \\ - $\quad$ Tipo de aficiones o hobbies que desempeñan los \\ trabajadores, entre otros (Henao, 2014).
}

Múltiples han sido los efectos que se atribuyen a la exposición al ruido, pero el más representativo, es el efecto fisiológico, el cual se deriva del uso de equipo instrumental, y generalmente su resultado es el deterioro de la audición, mientras que, los efectos psicológicos dependen de la respuesta subjetiva de las personas, misma que es recabada de un análisis estadístico para el estudio de los efectos denominados como: molestia, irritación, alteraciones del sueño, interferencia de la comunicación; en el caso de éste último para que exista una conversación moderada en la que la inteligibilidad sea de alrededor del $80 \%$ es necesario que el nivel de presión sonora de la conversación sea mayor a $12 \mathrm{dBA}$ con relación al ruido de fondo (Chávez, 2006).

Para obtener un entorno laboral saludable en el CCICEV, es necesario considerar ciertas medidas de prevención y control, para disminuir el nivel de ruido que perciben sus trabajadores.

Las medidas de control propuestas, permitirán reducir la exposición al ruido laboral a niveles aceptables, velando por la salud auditiva de las personas, y a su vez, se cumpliría la normativa ocupacional vigente, es decir, lo establecido en el Decreto Ejecutivo 2393 para Ecuador, en el que establece que, en caso de cumplir una jornada laboral de 8 horas, el nivel de presión sonora ponderado en A no debe ser superior a $85 \mathrm{dBA}$, y en los puestos de trabajo que demanden actividad intelectual, concentración o cálculo no debe exceder los 70 dBA (Presidencia de la República del Ecuador, 1986).

El ruido afecta a la salud, y se le considera perjudicial para cualquier área de trabajo, lo que a su vez, exige adoptar medidas de control ligadas a programas de vigilancia de la salud; el esfuerzo y compromiso es fundamental, ya que, al crear conciencia en el grupo de trabajo se consigue una cultura colectiva de seguridad (Delgado et al, 2010).

\section{METODOLOGÍA}

El CCICEV se encarga de evaluar la conformidad de los componentes o sistemas de los vehículos, mediante una simple inspección o ensayos de laboratorio, ya sea en proyectos de investigación, revisión técnica vehicular, laboratorio de emisiones, evaluación de vehículos nacionales e importados.

Las instalaciones que componen el CCICEV constan de un galpón y dentro de este se encuentran, el laboratorio de emisiones y revisión vehicular, dos bodegas, un mini comedor y las oficinas dispuestas en dos niveles como se observa en la Figura 1. Los puestos de trabajo del área administrativa desarrollan sus actividades en las oficinas, mientras que los puestos de trabajo del área técnica se desarrollan en el laboratorio, las oficinas y fuera de las instalaciones del CCICEV cuando la modalidad es por comisión de servicios. 


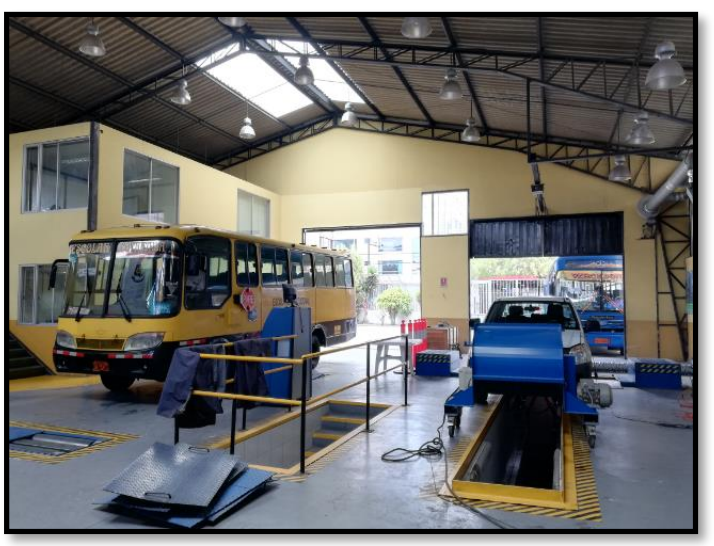

Figura. 1. CCICEV

Para establecer de forma clara y ordenada los pasos a seguir en la gestión de la exposición laboral al ruido, se eligió el modelo de gestión del riesgo aplicado al ruido laboral como se indica en la Figura 2 (Cortés, 2012).

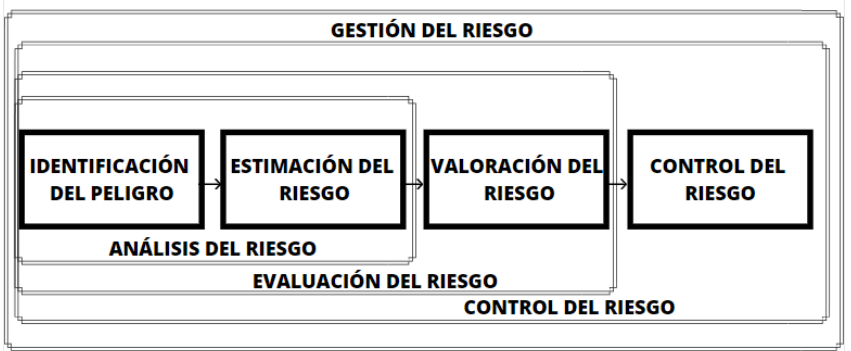

Figura. 2. Modelo de gestión del riesgo (Cortés, 2012)

Después de conocer las actividades que se desarrollan en el CCICEV, se comprobó la existencia de ruido laboral, y se llevó a cabo una investigación, cuyo caso particular, fue gestionar la exposición laboral al ruido, donde el nivel de medición y análisis de la información recabada fue cualitativa y cuantitativa, en cuyo caso las variables procedieron de un estudio no experimental que a su vez fue observacional, prospectivo, transversal y descriptivo. Además, el método empleado a lo largo de la investigación fue deductivo.

Según el Instituto Sindical de Trabajo, Ambiente y Salud (ISTAS, s.f.), el objetivo de identificar los peligros asociados al trabajo, consiste en:

- Eliminar los factores de riesgo fáciles de suprimir.

- Evaluar los riesgos que no es posible suprimir.

- Planificar y/o proponer la adopción de medidas de control.

\subsection{Identificación de los factores de exposición laboral al ruido}

Se empleó el método práctico basado en la evidencia, mediante una lista de verificación del "Manual para la evaluación y prevención de riesgos ergonómicos y psicosociales en PYME", debido a que el número de personas que laboran en el CCICEV es menor a 50 (INSHT, 2003a).

Luego, se solicitó la colaboración de las personas que conforman el CCICEV para llenar una encuesta de sondeo que permitiría identificar de forma cualitativa la incidencia del ruido laboral, la encuesta contó con 20 preguntas de opción múltiple para facilidad de diagnóstico. Con ello se obtuvo datos como: la jornada laboral, el área de trabajo, la permanencia laboral, edad, entre otros. La documentación histórica proporcionada por el CCICEV permitió identificar los puestos de trabajo, así como los servicios que ofrece y la demanda por año.

Posteriormente, los trabajadores fueron sometidos a exámenes médicos auditivos para conocer el estado de salud de sus oídos. Y se realizó la toma de datos mediante sonómetro para determinar el grado de exposición laboral a ruido.

\subsection{Evaluación de la exposición laboral al ruido}

El método de evaluación general de riesgos del INSHT, relaciona la probabilidad y consecuencia para determinar el nivel de riesgo, e interpretar el mismo de forma cualitativa, siendo así que la probabilidad puede llegar a ser baja, media o alta y éstas se pueden relacionar con una consecuencia en un rango que va desde ligeramente dañino a extremadamente dañino, dando como resultado un riesgo, trivial, tolerable, moderado, importante o intolerable (INSHT, 2000).

Y para la valoración cuantitativa, se debe emplear un método específico de exposición al ruido laboral, con el que sea posible establecer un plan de medición que permita una evaluación representativa y fiable.

De tal forma que la NTP 951, "Estrategias de medición y valoración de la exposición a ruido (II)" y el análisis de las condiciones de trabajo en el CCICEV permitieron determinar que la estrategia basada en el puesto de trabajo es adecuada para este proyecto, por su practicidad; además de ser considerada útil en situaciones en las que es complejo describir un patrón de trabajo (INSHT/INSST, 2012).

Después de identificar la estrategia de medición, fue importante obtener el nivel de presión sonora diario ponderado en A, para ello se empleó la Ecuación (2).

$$
L_{A_{e q, d}}=L_{A e q, T}+10 \log \left(\frac{T}{8}\right)
$$

Donde:

LAeq,d es el nivel de presión sonora diario equivalente ponderado en $\mathrm{A}$ [dBA].

LAeq, $T$ es el nivel de presión sonora continuo equivalente ponderado en $\mathrm{A}$ [dBA].

$\mathrm{T}$ es el tiempo de exposición en horas/día.

Y mediante la Tabla 1, se estableció el método de medición para ruido estable conforme al Real Decreto 286 de España, que considera el instrumento de medida necesario, el número de repeticiones y la duración de cada una según el tipo de ruido identificado. 
Tabla 1.Método de medición del Ruido

\begin{tabular}{|c|c|c|c|}
\hline Definición & $\begin{array}{l}\text { Instrumento } \\
\text { de medida }\end{array}$ & $\begin{array}{l}\text { Duración } \\
\text { por } \\
\text { medición }\end{array}$ & Número de mediciones \\
\hline $\mathrm{R}\left(\mathrm{L}_{\mathrm{Pa} * \mathrm{I}}\right)<5 \mathrm{~dB}$ & $\begin{array}{c}\text { Sonómetro, } \\
\text { Tipo } 2 \\
\text { (UNE- } \\
\text { EN60651:19 } \\
\text { 96)SLOW }\end{array}$ & $>=15 \mathrm{~s}$ & $\begin{array}{c}\mathrm{R} \leq 2 \mathrm{~dB} \Rightarrow>3 \mathrm{~m} \\
2 \mathrm{~dB}<\mathrm{R}<5 \mathrm{~dB}=>\geq 5 \mathrm{~m}\end{array}$ \\
\hline
\end{tabular}

El equipo de medición empleado fue un sonómetro Optimus con marca comercial Cirrus Research S.L., tipo CR161C cuyo número de serie es G061059, con un calibrador acústico cuyo nivel de calibración es $93.7 \mathrm{~dB}$, baterías y software Noisetools como indica la Figura 3.

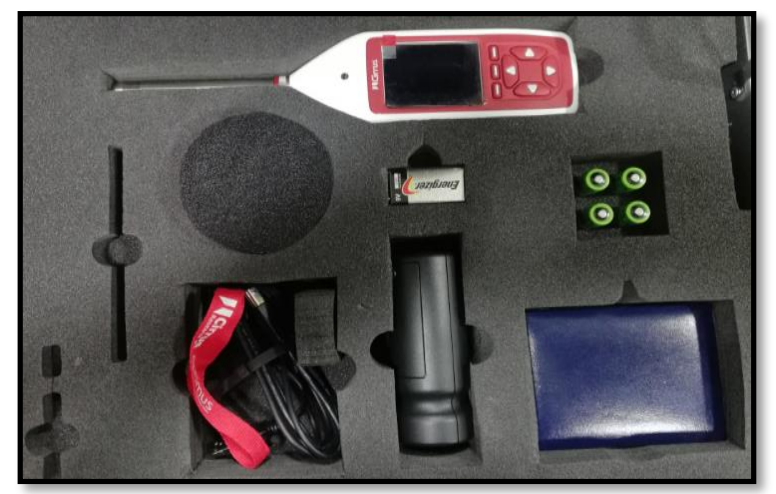

Figura. 3. Sonómetro e implementos utilizados para muestreos en el CCICEV

Una vez identificados los puestos de trabajo críticos, se entrevistó a las personas que se desenvuelven a diario allí, para determinar cuáles eran los puestos de trabajo y las actividades que desarrollan, como indica la Tabla 2.

Donde:

A1: Ensayo torque y potencia

A2: Ensayo lug down

A3: Ensayo ciclo europeo

A4: Ensayo opacidad

A5: Ensayo nivel sonoro tubo de escape

A6: Ensayo análisis de emisiones de gases

A7: Ensayo prueba de frenado

A8: Elaboración de informes, tareas de oficina

A9: Elaboración de informes, tareas de oficina

Tabla 2.Actividades desarrolladas por puesto de trabajo

\begin{tabular}{cc}
\multicolumn{2}{c}{ Área Administrativa } \\
\hline Puestos de Trabajo & Actividad \\
\hline Director Ejecutivo & A8, A9 \\
Especialista Administrativo & A8, A9 \\
Especialista Financiero & A8, A9 \\
Analista de Talento Humano & A8, A9 \\
Analista de Adquisiciones & A8, A9 \\
Auxiliar de servicios generales & A8, A9 \\
Contador & A8, A9 \\
Tesorero & A8, A9 \\
Secretaria & A8, A9
\end{tabular}

Tabla 2.Actividades desarrolladas por puesto de trabajo (Continuación) Área Técnica

\begin{tabular}{cc}
\hline Puestos de Trabajo & Actividad \\
\hline $\begin{array}{c}\text { Responsable Técnico de } \\
\text { Vehículos Nacionales }\end{array}$ & $(\mathrm{A} 8, \mathrm{~A} 9)^{*}$ \\
$\begin{array}{c}\text { Responsable Técnico de } \\
\text { Vehículos Importados }\end{array}$ & $(\mathrm{A} 8, \mathrm{~A} 9)^{*}$
\end{tabular}

Responsable Técnico de

Laboratorio de Emisiones y

Revisión Vehicular

Responsable de Calidad y

Acreditación
Inspector 1 de Vehículos

A1, A2, A3, A4, A5, A6, A7, A9 Nacionales

(A8, A9)*

$(\mathrm{A} 8, \mathrm{~A} 9)^{*}$

Inspector 1 de Vehículos Importados

Inspector 2 de Vehículos Nacionales

Inspector 2 de Vehículos Importados

$(\mathrm{A} 8, \mathrm{~A} 9)^{*}$

$(\mathrm{A} 8, \mathrm{~A} 9)^{*}$

(A8, A9)*

Técnico de Laboratorio $1 \quad$ A1, A2, A3, A4, A5, A6, A7, A8

$\overline{(\mathrm{A} 8, \mathrm{~A} 9)^{*} \text { Trabajadores que permanecen en las instalaciones del CCICEV }}$ cuando no desarrollan trabajo bajo la denominación comisión de servicios)

Con los datos antes recopilados, se determinó si existe sobreexposición tanto para el área técnica como para el área administrativa, para ello se compararon los valores calculados de nivel de presión sonora diario equivalente ponderado en A, con los valores límite, establecidos por el Decreto Ejecutivo 2393 los cuales no deben exceder los 85 dBA y para puestos de trabajo que demanden actividad intelectual no debe exceder los $70 \mathrm{dBA}$, siempre que la jornada laboral sea de 8 horas.

\subsection{Proposición de medidas de prevención y control de exposición laboral a ruido}

Las medidas de prevención y control fueron propuestas luego de analizar las condiciones de fuente, el medio y el receptor, de acuerdo a la jerarquía de control de riesgo según la norma ISO 45001 en la que se establece eliminación, sustitución, controles de ingeniería, señalización, alertas y/o controles administrativos y equipos de protección personal (ISO, 2018).

Existen dos fuentes significativas de ruido, una es el ventilador de enfriamiento, el cual es necesario para mantener controlada la temperatura del motor del vehículo durante la realización de los ensayos de laboratorio, como torque y potencia, lug down o ciclo europeo donde el vehículo se encuentra anclado mediante cadenas metálicas y aumenta su velocidad en el mismo sitio, de 0 a aproximadamente $120 \mathrm{~km} / \mathrm{h}$ en un tiempo inferior a $1 \mathrm{~min}$; y otra los automotores de prueba; es así que, para el ventilador de enfriamiento, se propuso encerrar el ruido mediante una cabina de insonorización, a más de ello se propuso absorber el ruido mediante paneles acústicos en el techo del galpón y revestir con material acústico la fachada frontal de las oficinas; en lo que se refiere a los controles administrativos, éstos se encontrarán directamente ligados a la reducción de la exposición así como a la formación e información, siendo un paso a favor el hecho de que ya existe señalización en el lugar de estudio; para los trabajadores del área técnica, se propuso el uso de equipo de protección auditiva, tipo tapón y tipo orejera. 


\subsection{Estimación de la atenuación efectiva}

Con la ayuda de la NTP 638 "Estimación de la atenuación efectiva de los protectores auditivos", se logró determinar la estimación de la atenuación efectiva de los protectores auditivos tipo orejera y tipo tapón; para el desarrollo de los cálculos se consideraron 11 protectores auditivos tipo orejera y 8 protectores auditivos tipo tapón, para así conocer de entre ellos cuáles podían ofrecer mayor atenuación tanto para sonidos graves como agudos.

En esta sección, se desarrollaron los tres métodos establecidos en la NTP 638, el método de las bandas de octava, el método de H, M y L y el método del SNR esto con el objetivo de determinar la reducción predicha del nivel de ruido (PNR) y el nivel de presión sonora efectivo ponderado en A (LA') para cada protector auditivo (INSHT, 2003b).

Debido a que el tiempo de utilización de un protector auditivo interviene directamente con la protección real que recibe una persona, se consideró el tiempo como variable; en cada uno de los cuatro protectores auditivos que registraron una mayor atenuación del ruido en el CCICEV.

\section{RESULTADOS Y DISCUSIÓN}

\subsection{Resultado de los exámenes médicos auditivos (audiometrías)}

De los exámenes médicos auditivos, se obtuvo como resultado que 1 de las 19 personas presenta hipoacusia bilateral, el diagnóstico de la audiometría indica que la persona de 36 años presenta una hipoacusia bilateral de moderada a severa, en la que se descarta una patología tubárica, pero es necesario el control de ruido, la persona debe acudir a control cada tres meses después de realizado el examen para determinar cuál ha sido su evolución.

\subsection{Resultado del método de evaluación general de riesgos del INSHT}

Mediante el desarrollo del método de evaluación general de riesgos del INSHT, en el que relaciona la probabilidad y consecuencia, se determinó la existencia de un riesgo importante que requiere ser controlado, las medidas pueden aplicarse en la fuente, medio y/o receptor, como indica la Tabla 3.

Tabla 3.Matriz de evaluación de riesgo físico por ruido

\begin{tabular}{ccccc}
$\begin{array}{c}\text { Lugar } \\
\text { de } \\
\text { Trabajo }\end{array}$ & $\begin{array}{c}\text { Identifi- } \\
\text { cación }\end{array}$ & Método & $\begin{array}{c}\text { Consecuen- } \\
\text { cia }\end{array}$ & $\begin{array}{c}\text { Probabi- } \\
\text { lidad }\end{array}$ \\
\cline { 2 - 5 } & (Riesgo) & Danación & \\
CCICEV & $\begin{array}{c}\text { Riesgo Físico } \\
\text { por ruido } \\
\text { (ensayos de } \\
\text { laboratorio en } \\
\text { automotores) }\end{array}$ & INSHT & Alta \\
\hline Riesgo & & Importante & \\
\hline
\end{tabular}

Tabla 3.Matriz de evaluación de riesgo físico por ruido (Continuación)

Medidas a Adoptar

Fuente Colocar una cabina para reducir el ruido del ventilador de enfriamiento.

Colocar paneles acústicos para reducir el ruido en el

Medio galpón.

Revestimiento de fachada de oficinas.

Receptor Uso de protección auditiva para atenuar el ruido en el oído de los trabajadores.

\subsection{Resultado de tabulación de datos obtenidos del sonómetro}

Posterior a la tabulación de los datos de nivel de presión sonora, se calculó el nivel de presión sonora equivalente ponderado en A y como resultado se obtuvo que cada actividad sobrepasa los $85 \mathrm{dBA}$ en laboratorio, y en oficina sobrepasa los $70 \mathrm{dBA}$ en 8 horas, lo que indica que la persona se encontraría sobreexpuesta si se realizaría una de estas actividades durante toda la jornada de forma continua, de acuerdo con lo que establece el Decreto Ejecutivo 2393, por ahora, estos datos permitirán conocer el nivel de presión sonora equivalente diario ponderado en $\mathrm{A}$.

Una vez identificadas las actividades que se realizan en los puestos de trabajo y luego de calcular su respectivo nivel de presión sonora equivalente ponderado en $\mathrm{A}$, se determinó el nivel de presión sonora equivalente diario ponderado en A, para cada puesto de trabajo, de acuerdo al tiempo en el que se lleva a cabo cada actividad.

De ello, se obtuvo como resultado que los dos puestos (técnico de laboratorio 1 y responsable técnico de laboratorio de emisiones y revisión vehicular) sobrepasan los $85 \mathrm{dBA}$ por jornada laboral de 8 horas, esto con base en el Decreto Ejecutivo 2393 y de acuerdo al límite de tolerancia recomendado por la OMS.

Lo expuesto anteriormente es posible observar en el ejemplo de cálculo para el Puesto de Responsable Técnico de Laboratorio de Emisiones y Revisión Vehicular, con la siguiente secuencia:

1.- Se identifican las actividades que componen el puesto de trabajo como se indican en la Tabla 2.

2.- Este puesto de trabajo se encuentra compuesto de ocho actividades, siete de ellas son ensayos que se ejecutan en el laboratorio de emisiones y revisión vehicular; la actividad restante se la realiza dentro de las oficinas para redactar informes o emitir proformas.

3.- El método de medición del ruido, empleado corresponde a la Tabla 1, en ella establece que se debe recabar entre tres y cinco mediciones, siempre que la relación entre ellas no supere los $5 \mathrm{~dB}$, en este caso la relación entre el máximo y mínimo valor de nivel de presión sonora total del grupo de 5 tomas de datos, no supera los $2 \mathrm{~dB}$. 
4.- Estas mediciones fueron obtenidas en el lapso de un mes entre agosto y septiembre de 2019, en días al azar con tomas de datos en la mañana y tarde, el nivel de presión sonora que resultó de la toma de datos se representó en bandas de octava por cada actividad.

5.- Se determina el nivel de presión sonora de la actividad 1 para el valor de banda de octava $31,5 \mathrm{~Hz}$ mediante el cálculo del promedio de los cinco valores de nivel de presión sonora obtenidos mediante el uso del sonómetro con la ayuda de la Ecuación 3.

$$
N P S_{A}=\frac{\sum_{A 1}^{A n} N P S_{i}}{n}
$$

Donde:

$\mathrm{NPS}_{\mathrm{A}}$ es el nivel de presión sonora por actividad [dB].

$\mathrm{NPS}_{\mathrm{i}}$ es el nivel de presión sonora por cada medición [dB]. $\mathrm{n}$ es el número de mediciones.

$$
\begin{gathered}
N P S_{A}=\frac{\sum_{A 1}^{A n} N P S_{i}}{n} \\
N P S_{A 1(31,5)}=\left(\frac{99,1+99,9+100,6+101,2+100,4}{5}\right) d B \\
N P S_{A 1(31,5 \mathrm{~Hz})}=100,2 d B
\end{gathered}
$$

El valor promedio obtenido pertenece a la banda de octava de $31,5 \mathrm{~Hz}$, el cálculo se debe realizar para cada banda de octava entre $31,5 \mathrm{~Hz}$ hasta $16000 \mathrm{~Hz}$.

6.- Se calcula el nivel de presión sonora total de la actividad 1 mediante la suma logarítmica de los niveles de presión sonora con los datos por banda de octava de acuerdo a la Ecuación 4.

Donde:

$$
N P S_{A 1}=10 \log \left[10^{\frac{N P S_{1}}{10}}+10^{\frac{N P S_{2}}{10}}+\ldots \ldots+10^{\frac{N P S_{n}}{10}}\right]
$$

$\mathrm{NPS}_{\mathrm{A} 1}$ es el nivel de presión sonora por actividad [dB].

$\mathrm{NPS}_{\mathrm{n}}$ es el nivel de presión sonora por banda de octava [dB].

$$
\begin{gathered}
N P S_{A 1}=10 \log \left[10^{\frac{100,2_{31,5}}{10}}+10^{\frac{98,263}{10}}+10^{\frac{95,9125}{10}}+10^{\frac{91,8_{250}}{10}}\right. \\
+10^{\frac{89,7500}{10}}+10^{\frac{88,8_{1 k}}{10}}+10^{\frac{86,7_{2 k}}{10}}+10^{\frac{82,6_{4 k}}{10}} \\
\left.+10^{\frac{79,38 k}{10}}+10^{\frac{62,316 k}{10}}\right] \\
N P S_{A 1}=104 \mathrm{~dB}
\end{gathered}
$$

7.- Se determina el nivel de presión sonora equivalente ponderado en A, mediante la adición entre el nivel de presión sonora promedio de cada banda de octava con su respectivo valor de corrección de ponderación en A como indica la Ecuación 5.

$$
L_{e q A 1(\text { B.o. })}=N P S_{A 1(\text { B.o. })}+\text { Aten } A_{\text {B.o. }}
$$

Donde:

Leq $_{\mathrm{A} 1(B . O .)}$ es el nivel de presión sonora equivalente ponderado en A, por actividad en cada banda de octava [dBA].

$\mathrm{NPS}_{\mathrm{A} 1 \text { (B.O.) }}$ es el nivel de presión sonora por actividad en cada banda de octava $[\mathrm{dB}]$.

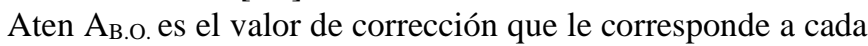
banda de octava de la red de ponderación A.

$$
\begin{gathered}
L_{\text {eqA1(31,5) }}=N P S_{A 1(31.5)}+\text { Aten } A_{31.5} \\
L_{e q A 1(31,5)}=[100,2+(-39,4)] d B A \\
L_{e q A 1(31,5)}=60,8 d B A
\end{gathered}
$$

8.- Se calcula el nivel de presión sonora equivalente ponderado en A total, de la Actividad 1 mediante la suma logarítmica de los niveles de presión sonora equivalentes ponderados en A de cada banda de octava como indica la Ecuación 6.

$$
L e q_{A 1}=10 \log \left[10^{\frac{L e q_{1}}{10}}+10^{\frac{L e q_{2}}{10}}+\ldots \ldots+10^{\frac{L e q_{n}}{10}}\right]
$$

Donde:

Leq $_{\mathrm{A} 1}$ es el nivel de presión sonora equivalente ponderado en A por actividad [dBA].

$\mathrm{Leq}_{\mathrm{n}}$ es el nivel de presión sonora equivalente ponderado en A por banda de octava [dBA].

$$
\begin{gathered}
L e q_{A 1}=10 \log \left[10^{\frac{60,831,5}{10}}+10^{\frac{72,0_{63}}{10}}+10^{\frac{80,0_{125}}{10}}+10^{\frac{83,2_{250}}{10}}\right. \\
+10^{\frac{86,5500}{10}}+10^{\frac{88,8_{1 k}}{10}}+10^{\frac{87,9_{2 k}}{10}}+10^{\frac{83,6_{4 k}}{10}} \\
\left.+10^{\frac{78,2_{8 k}}{10}}+10^{\frac{55,7_{16 k}}{10}}\right] \\
L^{L e q_{A 1}}=94 \mathrm{dBA}
\end{gathered}
$$

9.- Se realiza el mismo procedimiento desde el punto 5 al punto 8 con las actividades restantes que componen el puesto crítico 1 del área técnica, con los valores de nivel de presión sonora equivalente ponderado en A de cada actividad y el tiempo de duración de las mismas, luego se determina el nivel de presión sonora diario ponderado en A como se indica en la Ecuación 2.

$$
\begin{gathered}
L_{A_{e q, d}}=L_{A e q, T}+10 \log \left(\frac{T}{8}\right) \\
L_{A_{e q, d A 1}}=L_{A e q, A 1}+10 \log \left(\frac{T_{A 1}}{8}\right) \\
L_{A_{e q, d A 1}}=94 \mathrm{dBA}+10 \log \left(\frac{1 h}{8}\right) \\
L_{A_{e q, d A 1}}=85 \mathrm{dBA}
\end{gathered}
$$

10.- Luego de determinar el nivel de presión sonora equivalente diario ponderado en A de todas las actividades que se llevan a cabo en el puesto de trabajo, se determina el nivel de presión sonora equivalente diario total ponderado en A como indica la Ecuación 7. 
$L e q_{d T}=10 \log \left[10^{\frac{\operatorname{LeqdA_{1}}}{10}}+10^{\frac{\operatorname{LeqdA_{2}}}{10}}+\ldots \ldots+10^{\frac{\operatorname{LeqA_{n}}}{10}}\right]$

Donde:

Leq $_{\mathrm{dT}}$ es el nivel de presión sonora equivalente diario ponderado en $\mathrm{A}$ [dBA].

Leqd $_{\text {An }}$ es el nivel de presión sonora equivalente diario ponderado en A por actividad [dBA].

$$
\begin{gathered}
\operatorname{Leq}_{A 1}=10 \log \left[10^{\frac{85_{A 1}}{10}}+10^{\frac{81_{A 2}}{10}}+10^{\frac{81_{A 3}}{10}}+10^{\frac{77_{A 4}}{10}}\right. \\
\left.+10^{\frac{78_{A 5}}{10}}+10^{\frac{79_{A 6}}{10}}+10^{\frac{79_{A 7}}{10}}+10^{\frac{69}{10}}\right] \\
N P S_{A 1}=89 \mathrm{dBA}
\end{gathered}
$$

\subsection{Medida de control en la fuente}

La medida para reducir el ruido en la fuente, es aislar el ventilador de enfriamiento mediante una cabina de insonorización cuyas dimensiones son 2,40 m de largo, 1,00 m de ancho y $1,50 \mathrm{~m}$ de altura, con material de aluminio de $1 \mathrm{~mm}$ de espesor.

Con la cabina, el nivel de presión sonora equivalente ponderado en A de la máquina se vería reducido de $92 \mathrm{dBA}$ a $77 \mathrm{dBA}$ a $0,50 \mathrm{~m}$ de la misma y el nivel de atenuación total luego de considerar la pérdida por transmisión sería de 58 dBA aplicando adición de niveles sonoros entre las tres triangulaciones.

El ejemplo de cálculo que sustenta lo expuesto se encuentra a continuación:

1.- Se define el área que ocupará la cabina de insonorización a partir de sus dimensiones y los materiales que la conformarán.

\section{Dimensiones}

Largo (l) es 2,40 m

Ancho (a) es $1,00 \mathrm{~m}$

Altura (h) es $1,50 \mathrm{~m}$

\section{Materiales}

El área de aluminio se encontrará en las 3 caras laterales y la cara superior de la cabina, el área de hormigón, en la cara inferior o sección de losa donde se apoyará la cabina y la sección libre el área que permitirá la ventilación a la fuente de ruido. Para el cálculo se empleó las Ecuaciones 8, 9 y 10.

$$
\begin{aligned}
& A_{\text {aluminio }}=(l \times h)+(l \times a)+2 \times(a \times h) \\
& A_{\text {aluminio }}=(2,4 \times 1,50)+(2,4 \times 1,00)+2 \times(1,0 \times 1,50) \\
& =9,0 \mathrm{~m}^{2} \\
& A_{\text {hormigón }}=l \times a \\
& A_{\text {hormigón }}=2,4 \times 1,0=2,4 \mathrm{~m}^{2}
\end{aligned}
$$

$$
\begin{gathered}
A_{\text {aire }}=l \times h \\
A_{\text {aire }}=2,4 \times 1,5=3,6 \mathrm{~m}^{2}
\end{gathered}
$$

El área total es la sumatoria de áreas que forman parte en el diseño de la cabina como se observa en la Ecuación 11.

$$
\begin{aligned}
& A_{\text {total }}=A_{\text {aluminio }}+A_{\text {hormigón }}+A_{\text {aire }} \\
& A_{\text {total }}=9,0+2,4+3,6=15,0 \mathrm{~m}^{2}
\end{aligned}
$$

2.- Se identifican los vértices para la triangulación entre la fuente de ruido, el borde superior de una de las caras laterales o pantallas acústicas y el receptor.

3.- Se calcula el número de Fresnel $(\mathrm{N})$ mediante el uso de las Ecuaciones 12, 13, 14 y 15

$$
\begin{gathered}
N=\frac{2}{\lambda}(A+B-d) \\
\lambda=\frac{c}{f} \\
N_{c t e}=2(A+B-d) \\
N=\frac{f}{340} N_{c t e}
\end{gathered}
$$

Donde:

$\mathrm{N}$ : número de Fresnel

$\lambda$ : longitud de onda $[\mathrm{m}]$

c: velocidad de propagación del ruido en aire $[\mathrm{m} / \mathrm{s}]$

$\mathrm{f}$ : frecuencia $[\mathrm{Hz}]$

$\mathrm{N}_{\text {cte: }}$ : valor constante previo al cálculo donde interviene la frecuencia y la velocidad de propagación [m]

4.- Se identifica la pérdida por transmisión de cada material empleado en el diseño por banda de octava, además, se combinan en pares las áreas de los materiales que formarán parte de la cabina, de manera que $S_{1}>S_{2}$, siendo $S_{1}$ la sección de mayor área y $S_{2}$ la de menor área de los dos materiales considerados para el primer par de evaluación. Si se va a emplear más de dos materiales en el diseño de la cabina, entonces, la primera combinación cuenta como un material a combinarse con la sección del nuevo material que conformará el nuevo par de evaluación, y el procedimiento se mantiene hasta agotar todos los materiales que serán utilizados para la cabina de insonorización.

5.- Mediante la ayuda de ábaco para pérdida de aislamiento o curvas para el cálculo de la pérdida por transmisión resultante al considerar dos superficies de materiales distintos. (Cahueñas, 2018), se determina la pérdida de transmisión resultante.

6.- Se desarrolla el mismo procedimiento que para la triangulación vertical y se encuentran los valores del número de Fresnel por banda de octava, la pérdida por transmisión resultante y el nivel de atenuación que surge como resultado 
de la interposición de una pantalla acústica para las secciones triangulares horizontales.

\subsection{Medida de control en el medio de transmisión}

Otra medida que permite controlar el ruido es instalar 220 paneles acústicos de lana de vidrio con dimensiones de 2,45 m x 1,22 m x 0,08 m a dos caras, dispuestos en 5 hileras en la sección más corta del galpón, con un espacio entre ellos de $0,20 \mathrm{~m}$; cada hilera llevará 44 paneles separados a lo largo del galpón $0,40 \mathrm{~m}$.

Para determinar la absorción acústica de un recinto cerrado se debe partir de las áreas que lo componen los distintos materiales.

El coeficiente de absorción acústica varía porque depende de la frecuencia y el tipo de material, se expresa como indica la Ecuación 16, si el coeficiente de absorción acústica es 1, entonces la energía incidente es absorbida, pero si por el contrario, el coeficiente es 0 entonces toda la energía es reflejada.

$$
\alpha=\frac{E_{a}}{E_{i}}
$$

Donde:

$\alpha$ es el coeficiente de absorción acústica de un material

$\mathrm{E}_{\mathrm{a}}$ es la energía absorbida $[\mathrm{J}]$

$\mathrm{E}_{\mathrm{i}}$ es la energía incidente [J]

Mediante la Ecuación 17, se obtiene la absorción acústica de un material.

$$
A=S x \alpha
$$

Donde:

A es la absorción acústica $\left[\mathrm{m}^{2}\right]$ ó [Sabines]

$\mathrm{S}$ es la superficie del material $\left[\mathrm{m}^{2}\right]$

$\alpha$ es el coeficiente de absorción del material

Los coeficientes de absorción del material son valores distintos según el material.

Para determinar la absorción acústica de un recinto, se emplea la Ecuación 18.

$$
A=S_{1} x \propto_{1}+S_{2} x \propto_{2}+S_{3} x \propto_{3}+\cdots+S_{n} x \propto_{n}
$$

Donde:

A es la absorción acústica de un recinto [ $\left.\mathrm{m}^{2}\right]$ ó [Sabines]

$\mathrm{S}_{\mathrm{i}}$ es la superficie interior del recinto ocupada por un material $\mathrm{i}\left[\mathrm{m}^{2}\right]$

$\alpha_{\mathrm{i}}$ es el coeficiente de absorción del material i.

Mediante la Ecuación 19, es posible determinar el coeficiente de absorción promedio por material.

Donde:

$$
\bar{\alpha}=\sum_{i=1} \frac{s_{i} \times \alpha_{i}}{S}
$$

$\bar{\alpha}$ es el coeficiente de absorción promedio del material $\alpha i$ es el coeficiente de absorción del material i.

$\mathrm{Si}$ es la superficie interior del recinto ocupada por el material $\mathrm{i}\left[\mathrm{m}^{2}\right]$

$\mathrm{S}$ es la superficie interior total del recinto, ocupada por los materiales $\left[\mathrm{m}^{2}\right]$

A partir de la Ecuación 20, se obtiene la constante de cerramiento del recinto cerrado.

$$
R=\frac{s x \bar{\alpha}}{1-\bar{\alpha}}
$$

Donde:

$\mathrm{R}$ es la constante de cerramiento

$\mathrm{S}$ es la superficie interior total del recinto, ocupada por los materiales $\left[\mathrm{m}^{2}\right]$

$\bar{\alpha}$ es el coeficiente de absorción promedio del material

Para determinar el nivel de presión sonora cuando existe absorción acústica en los recintos, se emplea la Ecuación 21

$$
N P S=N W S+10 \times \log \left(\frac{Q}{4 \pi \times r^{2}}+4 / R\right)
$$

Donde:

NPS es el nivel de presión sonora [dB]

NWS es el nivel de potencia sonora $[\mathrm{dB}]$

$\mathrm{Q}$ es el coeficiente de direccionalidad

$\mathrm{r}$ es la distancia $[\mathrm{m}]$

$\mathrm{R}$ la constante de cerramiento

Se determina el nivel de presión sonora equivalente ponderado en A, a partir de la corrección de red de ponderación A, se determina la reducción de ruido por banda de octava con los valores de Noice Criteria por banda de octava; para el proyecto se consideró al CCICEV como un recinto tipo fábrica para ingeniería pesada, con un valor de Noice Criteria de 70; se determinó la reducción del ruido y el área equivalente de absorción, y por último el área de paneles requerida, desde el punto más cercano al más lejano de la fuente.

Mediante la Figura 4, es posible observar la reducción en la propagación del ruido cuando se considera los paneles acústicos (b), con relación a cuando no han sido considerados (a), para ello, la sección en rojo indica la exposición a ruidos mayores a $85 \mathrm{dBA}$, la sección en azul ruidos entre 80 y $85 \mathrm{dBA}$ y la sección en verde corresponde a ruidos inferiores a $80 \mathrm{dBA}$ para una jornada laboral de 8 horas.

Con la instalación de los paneles acústicos, el nivel de presión sonora ponderado en A disminuiría $5 \mathrm{dBA}$ a los $6 \mathrm{~m}$ y $8 \mathrm{dBA}$ a los $24 \mathrm{~m}$ de distancia, como indica la Figura 5. 


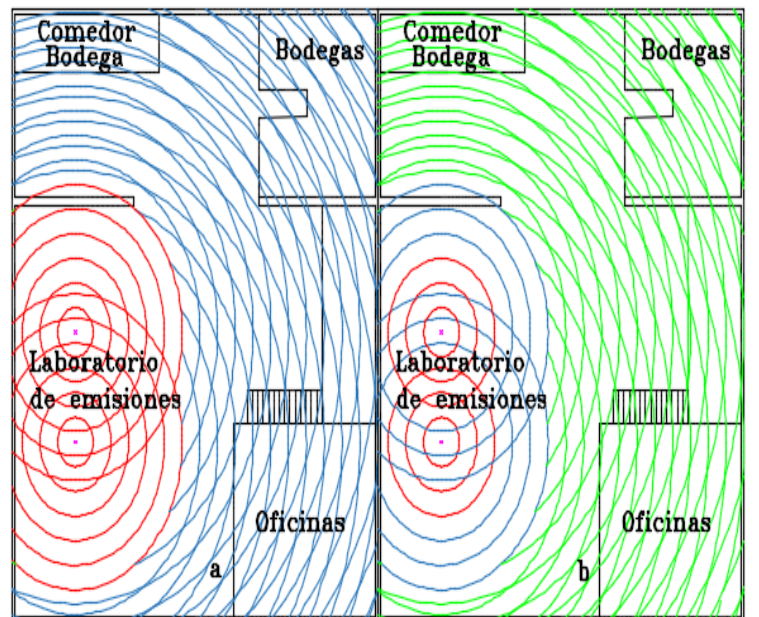

Figura. 4. Propagación del ruido con y sin paneles acústicos

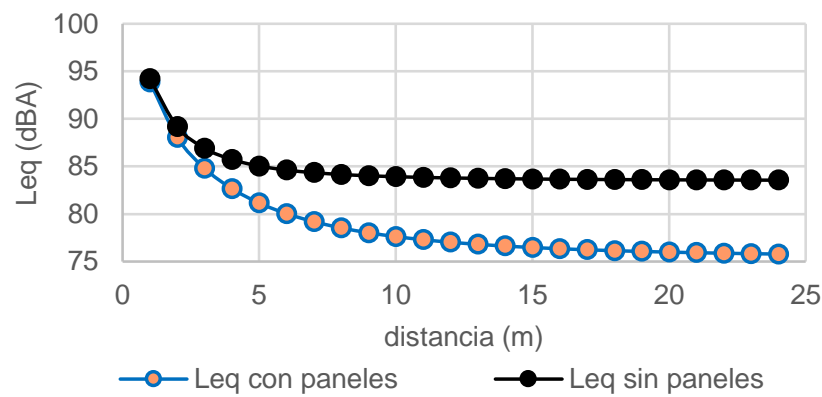

Figura. 5. Variación del nivel de presión sonora ponderado en A, con relación a la distancia de alejamiento de la fuente de ruido con y sin paneles acústicos

Para el caso del revestimiento de la fachada frontal de las oficinas del CCICEV, se consideró la combinación de materiales, aluminio de $1 \mathrm{~mm}$ y vidrio de $6 \mathrm{~mm}$; el nivel de presión sonora equivalente ponderado en $\mathrm{A}$ de las dos fuentes de ruido se reduciría de $94 \mathrm{dBA}$ a $75 \mathrm{dBA}$ a $10,0 \mathrm{~m}$ de la base y el nivel de atenuación total luego de considerar la pérdida por transmisión sería de $58 \mathrm{dBA}$ aplicando adición de niveles sonoros entre las tres triangulaciones, se utilizó el mismo principio que para el diseño de la cabina de insonorización, con el objetivo es evitar que el ruido interrumpa las actividades laborales en las oficinas del CCICEV.

La Tabla 4 representa el costo en dólares de las tres medidas de control propuestas, este costo puede llegar a variar conforme pasa el tiempo, por lo tanto es referencial.

Tabla 4. Costos referenciales para el desarrollo de las tres medidas de

\begin{tabular}{|c|c|c|c|c|c|}
\hline 1,0 & Materiales & Unid. & Cant. & P. Unitario & P. Total \\
\hline & $\begin{array}{l}\text { Pls Aluminio } \\
\text { liso }(1,22 \times 2,44)\end{array}$ & $\mathrm{u}$ & 40 & 37,10 & 1484,00 \\
\hline & $\begin{array}{c}1,0 \mathrm{~mm} \\
\text { Pls Vidrio } \\
(2,4 \times 3,6) 6,0 \\
\mathrm{~mm}\end{array}$ & $\mathrm{u}$ & 2 & 411,26 & 822,52 \\
\hline & $\begin{array}{l}\text { Paneles lana de } \\
\text { vidrio }(1,2 \mathrm{x} \\
24,0) 40,0 \mathrm{~mm}\end{array}$ & $\mathrm{~m}^{2}$ & 1354 & 2,42 & 3276,68 \\
\hline & Pernos & $\mathrm{u}$ & 3800 & 0,002 & 7,60 \\
\hline & Caucho & $\mathrm{m}$ & 1803 & 0,20 & 360,60 \\
\hline
\end{tabular}

Tabla 4. Costos referenciales para el desarrollo de las tres medidas de

\begin{tabular}{|c|c|c|c|c|c|}
\hline \multirow[t]{4}{*}{1,0} & Materiales & Unid. & Cant. & P. Unitario & P. Total \\
\hline & $\begin{array}{c}\text { Perfil Aluminio } \\
1,0 \times 0,04\end{array}$ & $\mathrm{~m}$ & 103 & 2,06 & 212,18 \\
\hline & $\begin{array}{c}\text { Perfil Aluminio } \\
1,0 \times 0,1\end{array}$ & $\mathrm{~m}$ & 1700 & 4,75 & 8075,00 \\
\hline & & & & Sub-total 1: & S/. 14.238,58 \\
\hline \multirow[t]{4}{*}{2,0} & Mano de Obra & Unid. & Cant. & P. Unitario & P. Total \\
\hline & Montador & $\mathrm{m}^{2}$ & 760 & 19,58 & 14880,80 \\
\hline & Ayudante & $\mathrm{m}^{2}$ & 760 & 12,20 & 9272,00 \\
\hline & & & & Sub-total 2: & S/. 24.152,80 \\
\hline \multirow[t]{4}{*}{3,0} & Mano de Obra & Unid. & Cant. & P. Unitario & P. Total \\
\hline & Herramientas & $\mathrm{m}^{2}$ & 760 & 7,96 & 6049,60 \\
\hline & & & & Sub-total 3: & S/. $6.049,60$ \\
\hline & & \multicolumn{2}{|c|}{ Total $(1-3): \$$} & & S/. $44.440,98$ \\
\hline
\end{tabular}

\subsection{Medida de control en el receptor}

Como resultado de la estimación de la atenuación efectiva de 19 protectores auditivos, 11 son tipo orejera y 8 son tipo tapón, se pudo determinar que los tapones 3M 1100 y 1110 ofrecen una mayor atenuación tanto para ruidos graves como agudos, como son tapones desechables pueden suponer una desventaja, por lo tanto también existe la opción de elegir los tapones 3M Ultrafit que ofrecen una segunda mejor atenuación con la ventaja de que no son desechables.

Si la preferencia es el uso de protectores auditivos tipo orejera que atenúen sonidos graves como agudos, se recomienda el uso del protector Sonis 3, pero si se trata de controlar los molestos sonidos agudos, el protector Sonis $\mathrm{C}$ ofrece una mayor atenuación, esto se determinó mediante los tres métodos de estimación de la atenuación efectiva de los protectores auditivos que proporciona la NTP 638.

De igual forma, de acuerdo a la misma NTP 638 indica que el método de bandas de octava para determinar tanto el PNR como L'A es fiable ya que considera el espectro de atenuación del protector auditivo . El siguiente método que brinda una buena aproximación en el cálculo del PNR es el método H, M y L, pero se debe tener cierto cuidado con el método SNR ya que este puede variar si preponderan frecuencias sumamente altas o excesivamente bajas en el espectro de atenuación del protector auditivo.

En cualquier caso, el uso de equipo de protección individual debe considerarse como la última medida de control, y esta debería ser de carácter complementario y temporal, además los trabajadores deben conocer y respetar las recomendaciones del fabricante en cuanto al correcto uso y mantenimiento según sea la elección del protector auditivo.

De acuerdo con las garantías que ofrece cada fabricante con relación al tiempo de uso del equipo de protección, se debe considerar de forma especial el hecho de que pueden presentarse riesgos derivados de la utilización del equipo de protección ya sea por mal uso o el uso excesivo del mismo. 


\subsection{Tiempo de utilización del protector auditivo}

El resultado de los niveles de atenuación según el tipo de protector auditivo y el tiempo de utilización del mismo, en los primeros 5 min de no utilización, presenta una variación en la atenuación global, esta disminuye alrededor de $10 \mathrm{dBA}$ con el uso de el protector auditivo tipo orejera Sonis $\mathrm{C}$ al igual que con los protectores auditivos tipo tapón 3M 1100/1110 y 3M Ultrafit, cosa que no ocurre con el protector tipo orejera Sonis 3 que disminuye aproximadamente $20 \mathrm{dBA}$.

Tabla 5. Nivel de presión sonora equivalente diario eficaz para los 4 tipos de protectores auditivos seleccionados de acuerdo a la atenuación

\begin{tabular}{ccccc}
$\begin{array}{c}\text { Tiempo de } \\
\text { utilización } \\
\text { del } \\
\text { protector }\end{array}$ & \multicolumn{5}{l}{ Nivel de atenuación Naten (dBA) } \\
\hline $\begin{array}{c}\text { Horas y } \\
\text { minutos }\end{array}$ & Sonis 3 & Sonis C & 3M & 3M \\
\hline Uh00 & 60 & 71 & 69 & 70 \\
7h25 & 80 & 80 & 79 & 80 \\
6h50 & 82 & 82 & 82 & 82 \\
6h15 & 84 & 84 & 84 & 84 \\
5h40 & 85 & 85 & 85 & 85 \\
5 h05 & 86 & 86 & 86 & 86 \\
0h00 & 89 & 89 & 89 & 89 \\
\hline
\end{tabular}

De acuerdo con la Tabla 5, la mayor variación por no usar el protector auditivo se da en los primeros $35 \mathrm{~min}$ si se considera el tiempo de forma global y 5 min al considerar cada actividad, al cumplir $1 \mathrm{~h} 10 \mathrm{~min}$ del no uso del protector auditivo considerado como 10 min por actividad, la variación de atenuación deja de ser notable entre los 4 protectores auditivos pero aún se mantienen dentro de una exposición aceptable, pero deja de suceder a las 2 h 20 min de no uso de los protectores auditivos, es decir, a los 20 min de no uso en cada actividad; para obtener estos resultados las actividades en laboratorio fueron consideradas con la atenuación de cada uno de los protectores auditivos y para la actividad en oficina, se mantuvo el valor de exposición determinada como la presión sonora equivalente ponderada en $\mathrm{A}$.

En lo que se refiere a las medidas administrativas $u$ organizativas, es necesario que los trabajadores realicen pausas activas o pasivas en lugares libres de ruido, que apaguen las fuentes de ruido si no están en funcionamiento y que informen a sus compañeros de trabajo cada vez que vayan a realizar una actividad ruidosa, esto con el objetivo de reducir la exposición laboral de los trabajadores.

También la señalización de los espacios de trabajo, la formación e información periódica referente a seguridad y salud laboral debe ser impartida a todos y cada uno de los trabajadores.

A su vez se debe llevar a cabo programas de vigilancia de la salud, donde prevalezcan los exámenes de inicio, y fin de gestión, así como, los exámenes periódicos; para llevar un registro histórico de los acontecimientos.

\section{CONCLUSIONES}

- Después de identificar los factores de exposición laboral al ruido se concluye que efectivamente el ruido es parte del día a día del personal del CCICEV, y que a más de percibirlo genera ciertas interrupciones y molestias mientras se desarrollan las actividades laborales.

- Como consecuencia del análisis cualitativo y cuantitativo de la evaluación de riesgos, se concluye que el riesgo es importante y el nivel de presión sonora equivalente diario ponderado en $\mathrm{A}$ en los dos puestos críticos del área técnica es de 89 dBA y en el puesto crítico del área administrativa es de $75 \mathrm{dBA}$ para una jornada laboral de 8 horas. Es decir, existe sobreexposición ya que sobrepasa los valores límite de 85 dBA y 70 dBA establecidos en el Decreto Ejecutivo 2393.

- Las medidas de control propuestas para la fuente y el medio de transmisión lograrían reducir el ruido significativamente; la cabina de insonorización de aluminio de espesor de $1 \mathrm{~mm}$, permitiría reducir el ruido de $92 \mathrm{dBA}$ a $77 \mathrm{dBA}$ a $0,50 \mathrm{~m}$ de la misma y el nivel de atenuación total luego de considerar la pérdida por transmisión sería de 58 dBA, los 220 paneles de lana de vidrio anclados al techo del galpón dispuestos a dos caras, permitirían reducir el ruido de $95 \mathrm{dBA}$ a $80 \mathrm{dBA}$ a los $6 \mathrm{~m}$ de distancia y a los $24 \mathrm{~m}$ se encontraría en $76 \mathrm{dBA}$, en el caso del revestimiento de aluminio de espesor $1 \mathrm{~mm}$ y vidrio de espesor $6 \mathrm{~mm}$ de la fachada frontal de las oficinas, disminuiría el ruido de $94 \mathrm{dBA}$ a $75 \mathrm{dBA}$ a $10,0 \mathrm{~m}$ de la base y el nivel de atenuación total luego de considerar la pérdida por transmisión sería de $58 \mathrm{dBA}$ aplicando adición de niveles sonoros entre las tres triangulaciones.

- Las medidas administrativas u organizativas como pausas activas o pasivas en un ambiente silencioso, apagar las fuentes de ruido si no se necesitan, informar a los presentes si se va a realizar una actividad ruidosa, la señalización, la formación e información así como la vigilancia de la salud, deben ser parte de la gestión del ruido, ya que, permiten reducir el tiempo de exposición de los trabajadores.

- Los protectores auditivos deben ser la última opción a considerar cuando de medidas de control se trata, esto debido a que es un elemento ajeno al cuerpo humano y resulta incómodo al usuario, entonces, si se logra implementar pronto las medidas antes descritas, solo se requeriría el uso de protectores auditivos en los primeros $3 \mathrm{~m}$ alrededor al foco de ruido como se muestra en la Figura 4, mientras dure la actividad 1 o las dos fuentes de ruido se encuentren funcionando al mismo tiempo.

- Para reducir la incidencia de ruido mientras se logra implementar las medidas de control en la fuente y en el medio de transmisión, se debe usar los protectores auditivos en todas las actividades de la 1 a la 7 , con lapsos de descanso por actividad, máximo de $15 \mathrm{~min}$, porque de no hacerlo la exposición diaria superaría los $84 \mathrm{dBA}$. 
- La eficacia de los protectores auditivos, tipo orejera Sonis 3 y Sonis $\mathrm{C}$ así como los protectores auditivos tipo tapón 3M 1100/10 y 3M Ultrafit brindan el mismo nivel de atenuación luego de los 70 min de no uso, donde el no uso se considera $10 \mathrm{~min}$ en cada actividad y no de forma seguida o continua, y la mayor eficacia de entre todos los protectores auditivos analizados, es el Sonis 3 siempre que no existan períodos de no uso durante la jornada laboral de 8 horas.

- El nivel de presión sonora equivalente diario de $89 \mathrm{dBA}$ que reciben los receptores de los dos puestos críticos del área técnica se vería reducido en $9 \mathrm{dBA}$ si utilizan los protectores auditivos durante el desarrollo de sus actividades en el laboratorio con períodos de descanso máximo de 5 min.

- Otro aspecto a tomar en cuenta es la distribución de ambientes (laboratorio - oficinas) porque el ruido distrae y molesta, cosa que impide desarrollar las actividades laborales administrativas de forma apropiada, de ser posible lo ideal sería reubicar las oficinas de tal manera que estas no se encuentren dentro del galpón donde se desarrollan las actividades de laboratorio, con ello el número de personas expuestas disminuiría y a su vez el personal técnico podría descansar en zonas libres de ruido.

\section{RECOMENDACIONES}

- Realizar un plan de prevención de riesgos laborales general, así como un plan de emergencias en el CCICEV, para evitar situaciones que puedan afectar a los trabajadores, visitantes, a las instalaciones y al medio ambiente velando así por la continuidad de actividades laborales.

- Establecer un programa de vigilancia de la salud en el que se realice exámenes médicos auditivos al inicio y fin de gestión, así como, de control periódico cada 3 años, para cumplir en cierta forma las medidas administrativas.

- Formar a los trabajadores mediante charlas relacionadas a seguridad en el trabajo para que puedan conocer los riesgos a los que se encuentran expuestos y así evitar actos o condiciones inseguras, siendo de igual forma parte primordial en cuanto al cumplimiento de las medidas administrativas.

- Realizar pausas activas y pausas pasivas de ser el caso, para que las personas puedan despejar su mente y reducir el estrés que puedan llegar a sentir.

- Generar buenas prácticas laborales en las cuales se comprometan los trabajadores de forma responsable con su seguridad y la de terceros.

- Implementar conductas de seguridad colectiva evitando conductas competitivas, esto permitirá el crecimiento del grupo de trabajo y la valoración de todos sus miembros.

- Evaluar la contaminación laboral por emisión de gases para futuras investigaciones en el CCICEV.

\section{REFERENCIAS}

Baraza, X., Castejón, E., y Guardino, X. (2014). Higiene Industrial (1era ed.). Barcelona, España: UOC.

Cahueñas, R. (2018). Diseño de una cabina insonorizada para un Laboratorio de calibración de equipos de Monitoreo de ruido. Obtenido de https://repositorio.uisek.edu.ec/bitstream/123456789/3177/2/TESI S\%20FINAL_RODRIGO\%20CAHUE\%C3\%91AS_AGOSTO\%2 02018.pdf. (Diciembre, 2019)

Chávez, J. (2006). Efectos del ruido sobre la salud. Ciencia y Trabajo. Obtenido http://sgpwe.izt.uam.mx/files/users/uami/patt/3._Contaminacion _Fisica/3_ruidoefectos.pdf (Diciembre 2019).

Cortés, J. (2012). Seguridad e higiene. (10a. ed.). Madrid, España: Tébar Flores.

Delgado, S., Ena, T., Ena, B., y Martin, B. (2010). Formación y orientación laboral (1era ed.). Madrid, España: Paraninfo.

Falagán, M. (2008). Higiene Industrial Agentes físicos y actividades especiales (2). Oviedo, España: Fundación Luis Fernández Velasco.

Henao, F. (2014). Riesgos físicos I ruido, vibraciones y presiones anormales (2da ed.). Bogotá, Colombia: Ecoe Ediciones.

INSHT NTP 270. (s/f). NTP 270. Evaluación de la exposición al ruido. Determinación de niveles representativos. Obtenido de https://www.insst.es/documents/94886/327166/ntp_270.pdf/9c674 732-ce77-481f-8c38-ffc03579bb75 (Agosto, 2019).

INSHT. (2000). Evaluación de riesgos laborales. Obtenido de https://www.insst.es/documents/94886/96076/Evaluacion_riesgos. pdf/1371c8cb-7321-48c0-880b-611f6f380c1d (Diciembre, 2019).

INSHT. (2003a). Manual para la evaluación y prevención de riesgos ergonómicos y psicosociales en PYME. Obtenido de: https://www.insst.es/documents/94886/96076/evaluacionriesgospy me/391f8fb1-d5dd-4a59-af90-b52d15d32633 (Agosto, 2019).

INSHT. (2003b). NTP 638 Estimación de la atenuación efectiva de los protectores auditivos. Obtenido de https://www.insst.es/documents/ 94886/326775/ntp_638.pdf/ec7cda7b-d636-48de-8d05020cd25857a9 (Agosto, 2019)

INSHT/INSST. (2012). NTP 951 Estrategias de medición y valoración de la exposición a ruido (II): tipos de estrategias. Obtenido de https://www.insst.es/documents/94886/326879/951w.pdf/fc57e51 d-5251-4662-ba16-e1b3a6a8706d (Agosto, 2019).

ISO. (2018). Norma Internacional ISO 45001. Sistemas de gestión de la seguridad y salud en el trabajo. Ginebra, Suiza: ISO

ISTAS, I. S. (s.f.). Evaluación de riesgos laborales. Obtenido de: https://istas.net/salud-laboral/actividades-preventivas/evaluacionde-riesgos-laborales. (Agosto, 2019).

Presidencia de la República del Ecuador. (1986). Decreto ejecutivo 2393: Reglamento de seguridad y salud de los trabajadores y mejoramiento del medio ambiente de trabajo. Obtenido de: http://www.trabajo.gob.ec/wpcontent/uploads/downloads/2012/12 /Reglamento-de-Seguridad-y-Salud-de-los-Trabajadores-yMejoramiento-del-Medio-Ambiente-de-Trabajo-DecretoEjecutivo-2393.pdf. (Enero, 2020).

Suter, A. (2012). Capítulo 47. Ruido. Obtenido de https://www.insst.es/documents/94886/162520/Cap\%C3\%ADtulo +47.+Ruido (Agosto, 2019). 


\section{BIOGRAFÍAS}

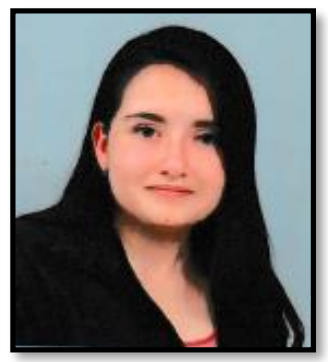

Gisela Carrera, Ingeniera Civil mención Estructuras, por la Escuela Politécnica Nacional en el 2016, Magister en Seguridad Industrial, mención Prevención de Riesgos Laborales, por la Escuela Politécnica Nacional en el 2020, se ha desempeñado como Asistente de Laboratorio de Ensayos, Asistente Técnico de Obras Públicas, Supervisora de Proyectos, Profesora Ocasional a tiempo completo, Técnico Docente Politécnico a tiempo completo y actualmente se desempeña como Consultora de Obras Civiles.

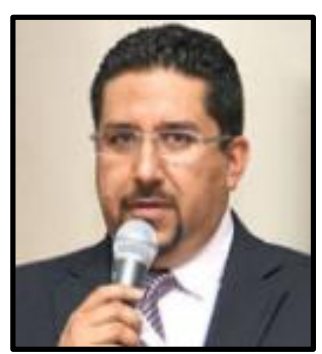

Francisco Salgado, Ingeniero Químico, por la Escuela Politécnica Nacional en el 2002, Magister Europeo en Energías Renovables por la Universidad de Zaragoza en el 2008, Especialista en Radioquímica y Aplicaciones Nucleares, cursó sus estudios de Doctorado en la Universidad Nacional de San Martín de Argentina, fue Responsable de la Maestría de Seguridad Industrial de la Escuela Politécnica Nacional, fue Docente de la Facultad de Ingeniería Química y Agroindustrial y la Universidad SEK del Ecuador, dejó un gran legado a cada uno de sus estudiantes, actualmente es parte de los buenos recuerdos de cada una de las personas que pasaron a lo largo de su vida.

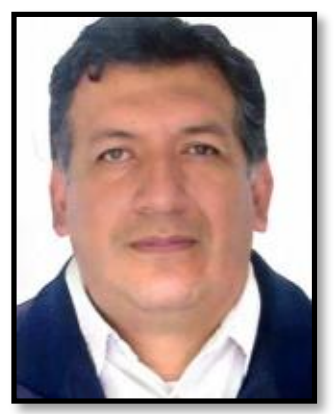

William Villacis, Doctorado de la Universidad de Buenos Aires área Ingeniería (en curso). Especialista en Seguridad Nuclear por la Universidad de Buenos Aires (2019). Especialista en Protección Radiológica y Seguridad de las Fuentes de Radiación por la Universidad de Buenos Aires (2017). Magíster en Seguridad Industrial y Salud Ocupacional por la Escuela Politécnica Nacional (2013). Ingeniero Químico por la Escuela Politécnica Nacional (2004). Se desempeña como docente universitario en las áreas de Higiene Industrial 2 y Fundamentos de Prevención de Riesgos Laborales para posgrado y Seguridad Industrial y Salud Ocupacional para pregrado en la Facultad de Ingeniería Química y Agroindustria. En la actualidad trabaja como Docente, Oficial de Seguridad Radiológica, Presidente Suplente del Comité de Seguridad e Higiene del Trabajo por parte del empleador en la Escuela Politécnica Nacional y es Coordinador de la Maestría en Seguridad Industrial Mención Prevención de Riesgos Laborales. 